\title{
Ultra-long-working-distance spectroscopy of single nanostructures with aspherical solid immersion microlenses
}

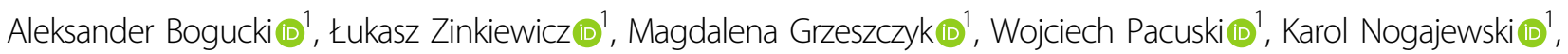

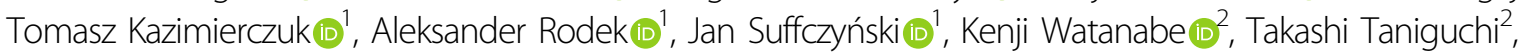 \\ Piotr Wasylczyk [1]', Marek Potemski iD, ${ }^{1,3}$ and Piotr Kossacki id
}

\begin{abstract}
In light science and applications, equally important roles are played by efficient light emitters/detectors and by the optical elements responsible for light extraction and delivery. The latter should be simple, cost effective, broadband, versatile and compatible with other components of widely desired micro-optical systems. Ideally, they should also operate without high-numerical-aperture optics. Here, we demonstrate that all these requirements can be met with elliptical microlenses 3D printed on top of light emitters. Importantly, the microlenses we propose readily form the collected light into an ultra-low divergence beam (half-angle divergence below $1^{\circ}$ ) perfectly suited for ultra-longworking-distance optical measurements (600 $\mathrm{mm}$ with a 1-inch collection lens), which are not accessible to date with other spectroscopic techniques. Our microlenses can be fabricated on a wide variety of samples, including semiconductor quantum dots and fragile van der Waals heterostructures made of novel two-dimensional materials, such as monolayer and few-layer transition metal dichalcogenides.
\end{abstract}

\section{Introduction}

Efficient light delivery to and collection from microoptical systems, in particular light emitters, is of paramount importance for their application potential and performance. It has been approached in many different ways: by placing mirrors beneath the light emitters, coating the substrate surface with anti-reflective layers to reduce internal reflection or shaping the transparent casing into the form of lenses, mesas, gratings or nanowires $^{1-10}$. These approaches help increase the critical angle of total internal reflection and/or reduce the Fresnel reflections at the interface. In the case of semiconductor nanostructures, solutions relying on solid immersion lenses (SILs) fabricated on top of the emitter, typically in

\footnotetext{
Correspondence: Aleksander Bogucki (Aleksander.Bogucki@fuw.edu.pl) or Piotr Kossacki (Piotr.Kossacki@fuw.edu.pl)

${ }^{1}$ Faculty of Physics, University of Warsaw, ul. Pasteura 5, 02-093 Warsaw, Poland ${ }^{2}$ National Institute for Materials Science, Tsukuba, Ibaraki 305-0044, Japan Full list of author information is available at the end of the article
}

the shape of a hemisphere, are frequently used. SILs manufactured with 3D subtractive techniques, such as electron beam lithography or focused ion beam, can increase the photon extraction up to $23 \%$ or even further up to $40 \%$ by employing additional optical structures ${ }^{7,11}$. None of these methods, however, have turned out to be fully satisfactory and free from considerable disadvantages: they still require additional light-collection optics of a high numerical aperture or cannot be used with atomically thin semiconductors, such as transition metal dichalcogenides. Therefore, efficient light collection from micro/nanoemitters and beam shaping (collimation) still present challenges in photonics and optical nanotechnology.

Once the light is extracted from the emitter, it must be shaped into a beam and directed to a measurement setup or another unit in an integrated electro-optical circuit. For industrial millimetre-scale light-emitting devices, such as LEDs, this problem has many well-established solutions, 


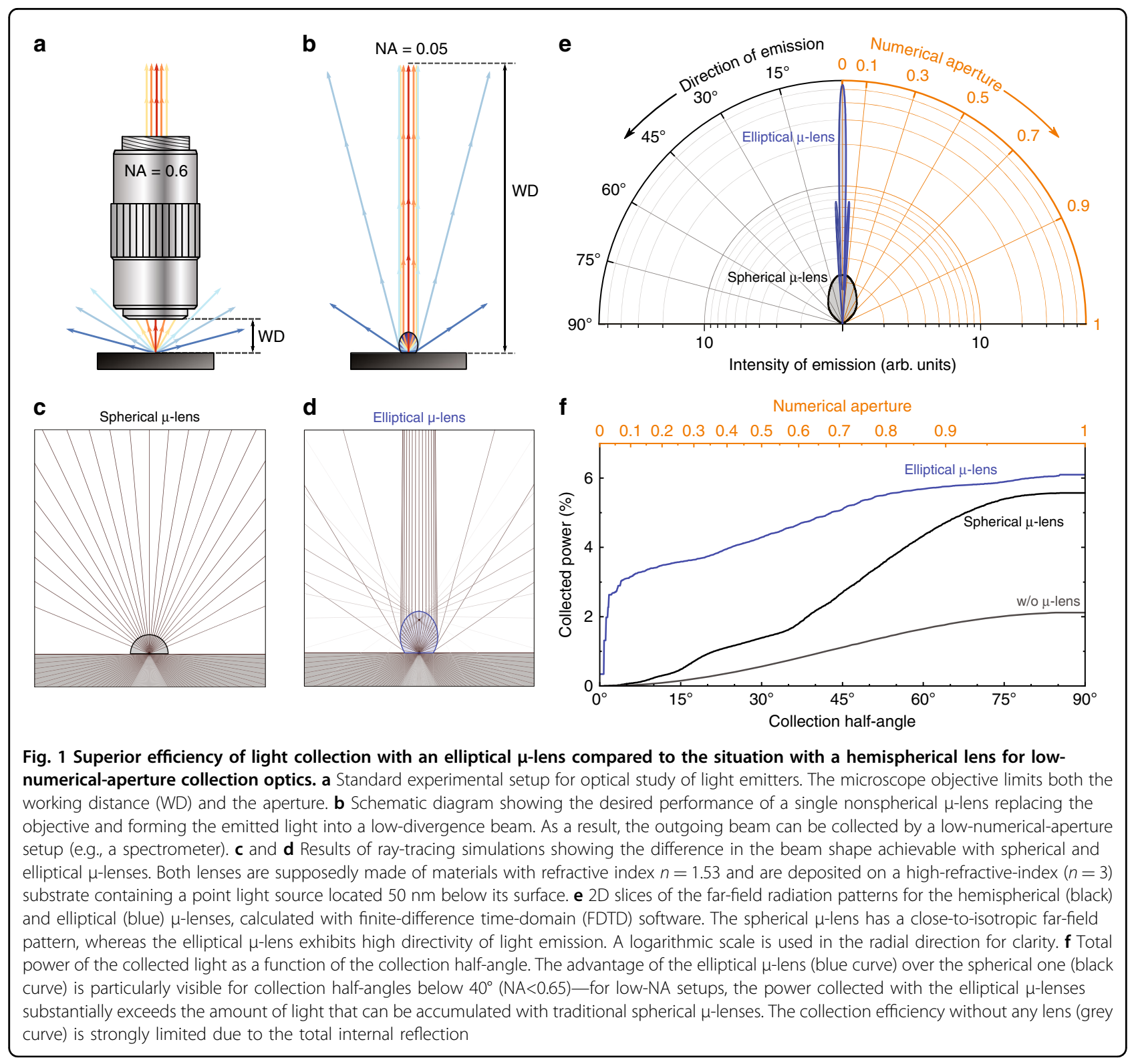

e.g., free-form lenses or dielectric total internal reflecting concentrators (DTIRCs) $)^{12-14}$. On the other hand, for prototype nanoscale emitters, high-numerical-aperture (NA) objectives (see Fig. 1a) are commonly used, resulting in a trade-off between the brightness and working distance (WD). In many experiments, however, the lens cannot be set close enough to the sample, and a significant amount of light is lost. These experiments, in particular, include those at cryogenic temperatures, in high continuous or pulsed magnetic fields, and with microwave or terahertz radiation as well as efficient light coupling into fibres for photonic lab-on-chip applications.

Surprisingly, the issues of light extraction and beam forming for nanoscale emitters are usually still treated separately, even though SILs are frequently manufactured by direct laser writing (DLW) - a versatile technique capable of printing lenses of virtually any shape. To our knowledge, the only approach to date for simultaneously addressing both problems for nanoscale point emitters has been to employ an evolutionary algorithm for light extraction from a nanowire, designed specifically to minimise the footprint of the structure ${ }^{15}$.

In this article, we present broadband elliptical microlenses ( $\mu$-lenses) fabricated by DLW, which enable spectroscopic measurements of single light emitters with extremely low-NA collection optics (see Fig. 1b). In a standard realisation of DLW-printed $\mu$-lenses, the observed increase in the light extraction efficiency is due to a reduction of the Fresnel reflections and an increase in the critical angle of total internal reflection ${ }^{16,17}$. Here, in 
addition to doubling the number of photons collected from the sample, the use of a nonspherical $\mu$-lens results in forming the collected light into an ultra-low-divergence beam (measured beam divergence half-angle smaller than $1^{\circ}$ ). Thus, the emitted light can be directly introduced into the collection optics with an effective WD of $\sim 600-700 \mathrm{~mm}$, which is 70 times longer than that of standard high-NA long-WD microscope objectives.

We demonstrate the performance of our $\mu$-lenses for two types of semiconductor emitters: (i) heterostructures based on monolayer transition metal dichalcogenides and hexagonal boron nitride (h-BN) and (ii) self-assembled quantum dots (QDs). The $\mu$-lens design works well for a broad spectral range spanning the visible and nearinfrared bands.

\section{Results}

\section{Optimum lens shape design}

Thus far, the simplest and most common form of the $\mu$ lens has been a hemisphere placed above a nanoscale light emitter $^{2,16-21}$. Usually, it is made of a material with a refractive index between that of the surrounding medium (low) and that of the sample (high), which not only enables an increase in the critical angle of total internal reflection but also significantly reduces the losses caused by the Fresnel reflections. The distribution of light leaving the hemispherical lens is isotropic (see Fig. 1c), and a microscope objective with a high NA is still required for efficient light collection.

Ideally, the light collection structure should (i) minimise the Fresnel reflections, (ii) be able to send the outgoing light towards distant collection optics and (iii) allow for fabrication on any sample/substrate. To achieve this with a single lens, it has to transform the homogeneous angular distribution of the photoluminescence (PL) from a point emitter into a collimated beam (see Fig. 1d). Within the approximation of geometric optics, the optimal lens shape when the refractive indices of the lens and substrate match is a Cartesian oval-an ellipsoid of revolution, known for almost four centuries ${ }^{22}$. To obtain a collimated beam, the emitter should be placed at the focal point situated further from the upper apex. The relation between the shorter half-axis $a$ of the ellipsoid and the longer half-axis $b$ (see Supplementary Information) is given by

$$
b=\frac{a n_{1}}{\sqrt{n_{1}^{2}-n_{0}^{2}}}
$$

where $n_{0}$ is the refractive index of the surrounding medium (air) and $n_{1}$ is the refractive index of the material the ellipsoid is made of (in our case, 1.53) ${ }^{23}$.

In general, the emitter may be embedded in a highrefractive-index substrate $\left(n_{2}\right)$ and located at a distance $d$ below its surface. To a first (paraxial) approximation, for $d<<b$, the lens still takes on the form of an elongated spheroid, cut on the bottom side by the substrate, while the presence of the substrate defines the apparent position of the emitter according to Snell's law (see Fig. 2a). In this case, the lens height (the distance between the ellipsoid top and the substrate surface) is given by

$$
h=a \sqrt{\frac{n_{1}+n_{0}}{n_{1}-n_{0}}}-d \frac{n_{1}}{n_{2}} .
$$

It is worth noting that under these conditions, the $\mu$ lens centre is no longer positioned at the substrate surface. This simple modification combined with a change of the well-established hemispherical design of a lens into an elliptical one offers a solution for both light extraction from the sample and light redirection towards the collection optics.
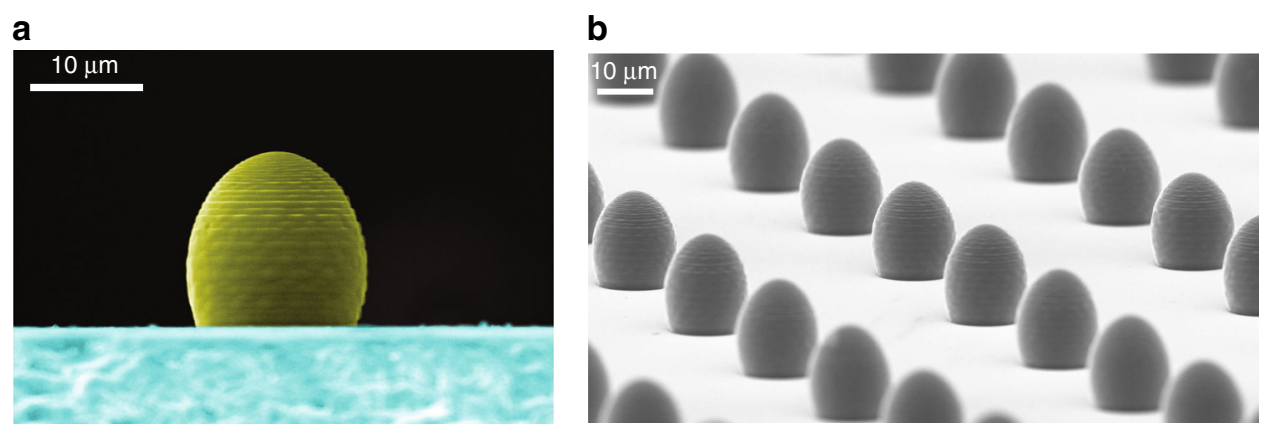

Fig. 2 Scanning electron microscope (SEM) images of 3D-printed $\mu$-lenses. a Side view of a $\mu$-lens (coloured in yellow) fabricated on a glass substrate (coloured in blue). Regardless of the residual roughness of the produced structures, the presented optical quality is more than sufficient for obtaining a well-collimated light beam. $\mathbf{b}$ Series of $\mu$-lenses distributed in a regular way across a substrate containing randomly dispersed light emitters, e.g., quantum dots. Due to the short printing time, it is possible to produce hundreds of $\mu$-lenses on the substrate surface and search for interesting light emitters afterwards. A regular grid of lenses also provides a convenient coordinate system for marking the positions of individual light sources. This enables moving the sample with a selected emitter to a different laboratory and measuring the same emitter in various experimental setups 
The above approach works well in the limit of geometric optics and allows for an arbitrary size of a $\mu$-lens. However, to obtain a beam with the lowest possible divergence, the analysis must be done in the framework of wave optics ${ }^{24,25}$. We have approached this by solving Maxwell's equations with a finite-difference time-domain (FDTD) method. Here, the maximum achievable WD for a given NA is determined by the minimal diameter of the $\mu$-lens-the smaller the lens diameter is, the larger the divergence of the outgoing beam. For a 1-inch lens placed $\sim 0.5 \mathrm{~m}$ from the sample, the minimum diameter of a $\mu$ lens should be $\sim 15 \mu \mathrm{m}$.

Figure 1e shows the simulated intensity of light emitted at different angles from a point source located $50 \mathrm{~nm}$ below the surface of a high-refractive-index $(n=3)$ semiconductor. The distribution of light emitted from an elliptical lens is highly directional (blue curve), in contrast to that from a hemispherical lens (black curve), which is close to isotropic. Figure 1f presents the integrated light intensity as a function of the collection angle. The advantage of the elliptical shape over the spherical shape is particularly visible for collection angles below $40^{\circ}$ (NA $<0.65)$. For small numerical apertures $(\mathrm{NA}<0.1)$, the increase in the intensity exceeds two orders of magnitude.

\section{Lens fabrication}

$\mu$-lenses were produced with two-photon 3D photolithography (DLW), which has been proven to yield highquality optical elements of arbitrary shape for lab-on-chip and optical fibre applications ${ }^{26-30}$. Figure $2 \mathrm{a}$ shows a scanning electron microscope (SEM) image of an elliptical $\mu$-lens with a diameter of $15 \mu \mathrm{m}$. Owing to the short printing time $(<4 \mathrm{~min}$ for a standard 3D lithography system with piezoelectric stages; see the "Methods" section for details), it is possible to manufacture hundreds of lenses, each covering a small portion of the sample surface, and to search for the emitters of interest afterwards. A grid of lenses (see Fig. 2b) provides a coordinate system for recording the emitter positions. This nondeterministic approach significantly simplifies the manufacturing procedure. Alternatively, high-accuracy deterministic positioning of $\mu$-lenses can be performed when the location of an emitter of interest is known or the emitter is visible under an optical microscope $\mathrm{e}^{7,16}$.

\section{Applications in spectroscopy}

To demonstrate the performance of the $\mu$-lenses, we tested them for two different semiconductor systems: selfassembled QDs and van der Waals heterostructures made of novel quasi-two-dimensional materials, i.e., monolayers of semiconducting transition metal dichalcogenides (S-TMDs) and h-BN. From the viewpoint of DLW-based $3 \mathrm{D}$ printing, these represent two very distant areas in the field of solid-state physics and technology.
The QDs, which are nanometre-sized structures formed during epitaxial growth of lattice-mismatched materials, are typically buried under a capping layer that insulates them from the detrimental influence of oxygen and water vapour in the air. Moreover, they are firmly linked to the surrounding host material. For the S-TMD-based van der Waals heterostructures, which are typically weakly bound to the supporting substrate, contact with just the ambient atmosphere and the contaminants it contains, as well as with many other chemicals can destroy them or irreversibly alter their properties. As a result, 3D printing of $\mu$ lenses over such objects constitutes a real challenge that, to the best of our knowledge, has not been undertaken thus far.

The interest in monolayer and few-layer S-TMDs and other 2D materials results from their unique electronic and optical properties ${ }^{31}$ as well as their mutual compatibility, which opens up the possibility of stacking them to form artificial crystals with tailored properties ${ }^{32}$. The STMDs, with a non-zero bandgap, are particularly well suited for optical applications, including optoelectronic sensors and light emitters ${ }^{33-38}$. We decided to use two popular representatives of the S-TMD family, namely, monolayer $\mathrm{WSe}_{2}$ and monolayer $\mathrm{MoSe}_{2}$. We demonstrate that additive DLW of polymer structures on top of STMD-based and h-BN-based heterostructures enhances the collection efficiency without deteriorating their optical properties. Figure 3b, c, e, f show optical microscope images of heterostructures consisting of a single layer of $\mathrm{WSe}_{2}$ or $\mathrm{MoSe}_{2}$ covered with a thin flake of h-BN deposited on a piece of a $90 \mathrm{~nm} \mathrm{SiO}_{2} / \mathrm{Si}$ substrate before and after printing the $\mu$-lenses. The characteristic lateral dimensions of the S-TMD monolayers are below $10 \mu \mathrm{m}$ to ensure that they can be approximated as point-like light sources. Figure 3a, d display room-temperature PL spectra collected without an external microscope objective (see the "Methods" section for details) for the h-BN/WSe $\mathrm{Wnd}_{2}$ and $\mathrm{h}-\mathrm{BN} / \mathrm{MoSe}_{2}$ heterostructures without (grey curve) and with (blue curve) the elliptical $\mu$-lenses. Both spectra were acquired with a lens of $f=500 \mathrm{~mm}$ and a 1 -inch diameter. The enhancement of the collection efficiency with the elliptical $\mu$-lens reaches $\times 450$ for the $\mathrm{h}$-BN/WSe ${ }_{2}$ heterostructure, while for the $\mathrm{h}-\mathrm{BN} / \mathrm{MoSe}_{2}$ heterostructure, the enhancement is approximately $\times 15$. The difference between these numbers can be explained by the fact that the dimensions of the monolayer flakes of $\mathrm{WSe}_{2}$ and $\mathrm{MoSe}_{2}$ are not the same, while the diameter of the $\mu$-lens is fixed at $70 \mu \mathrm{m}$. Since the $\mathrm{WSe}_{2}$ monolayer is a few times smaller than the $\mathrm{MoSe}_{2}$ monolayer, it better satisfies the approximation of the point-like emitter, for which the elliptical $\mu$-lens was designed.

The second test structure contains self-assembled QDs, which attract interest due to their remarkable optical properties, such as single photon emission or entangled 


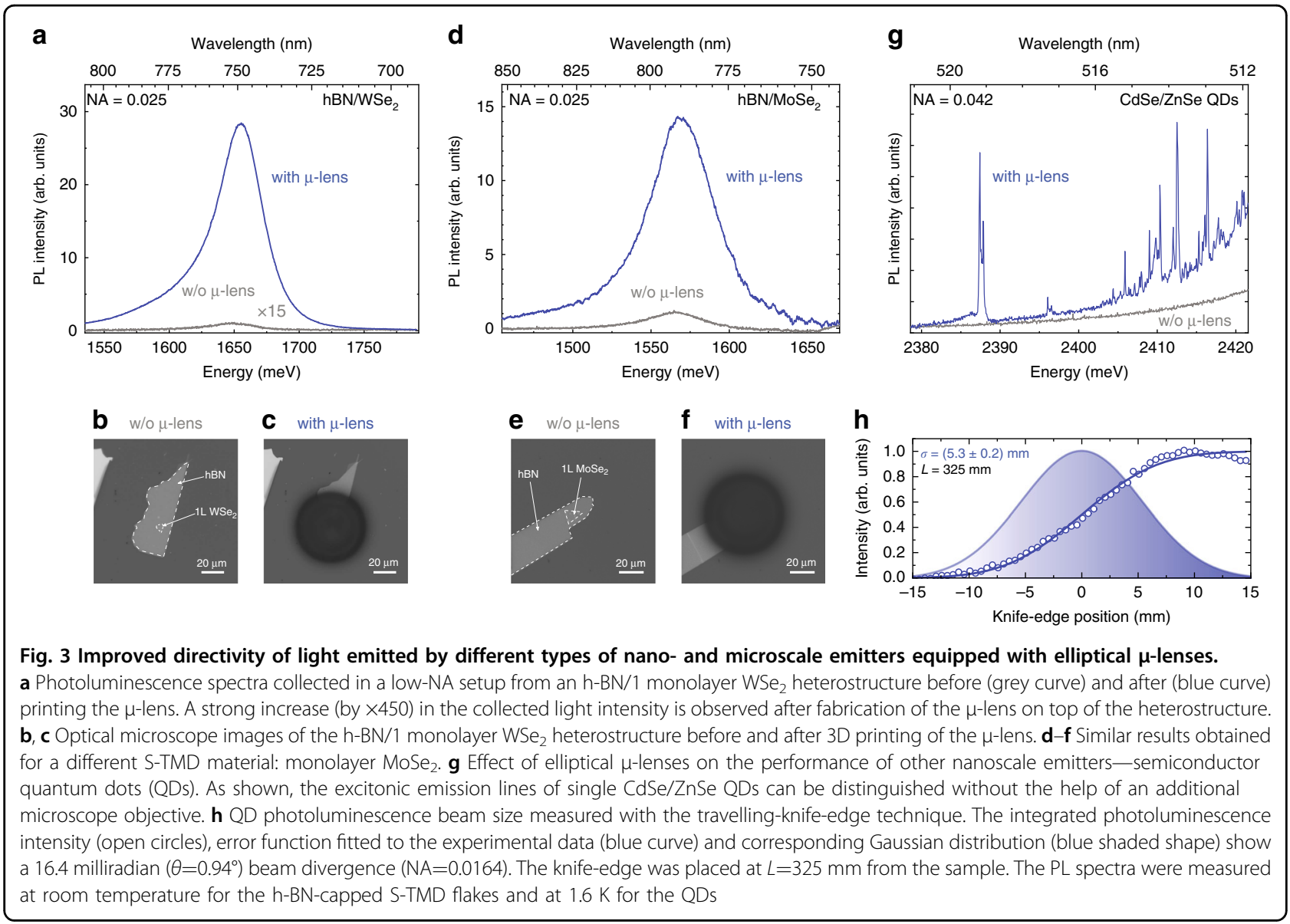

photon pair generation ${ }^{1,2,4,11}$. This system is a better model of a point-like light source. In contrast to the $h$ $\mathrm{BN} / \mathrm{S}$-TMD heterostructures, where single $\mu$-lenses were fabricated in specific areas determined by the heterostructure locations, here, we produced a large-scale array of $1400 \mu$-lenses (each $15 \mu \mathrm{m}$ in diameter) on a sample containing either $\mathrm{CdSe} / \mathrm{ZnSe}$ QDs emitting in the $500-520 \mathrm{~nm}$ range or $\mathrm{CdTe} / \mathrm{ZnTe}$ QDs emitting in the $600-650 \mathrm{~nm}$ range. All QDs are located $50 \mathrm{~nm}$ below the sample surface (more details are given in the Supplementary Information).

Figure 3g shows PL spectra of CdSe/ZnSe QDs collected without a microscope objective from a sample without a $\mu$ lens (grey curve) and with an elliptical $\mu$-lens (blue curve). The spectrum measured without the $\mu$-lens does not show any individual emission lines. Under the excitation laser spot ( $28 \mu \mathrm{m}$ in diameter), there are $\sim 10^{4}$ QDs emitting at different wavelengths. As a result, many individual sharp lines merge into a nearly uniform PL spectrum.

On the other hand, the laser beam impinging on the elliptical $\mu$-lens is focused to a spot with a diameter below $1 \mu \mathrm{m}$ (close to the diffraction-limited size) and thus excites much fewer QDs. Moreover, among all the excited QDs, only those satisfying the collimation conditions efficiently contribute to the collected PL spectrum, and sharp emission lines of individual QDs are clearly visible. The intensity gain in this case is estimated to be approximately 100 (see the "Methods" section for details).

We note that manufacturing $\mu$-lenses over the QDs did not visibly affect their properties. Figure $4 a, d$ show the PL spectra of a single CdTe/ZnTe QD and a single CdSe/ $\mathrm{ZnSe}$ QD coupled to a $\mu$-lens. The spectra feature wellresolved emission lines corresponding to recombination of a neutral exciton (X), a charged exciton (CX) and a biexciton (XX), consistent with the emission of QDs in unstructured samples ${ }^{39,40}$.

\section{Beam shape characterisation}

To measure the resulting PL beam size and divergence, we used the travelling knife-edge technique ${ }^{41}$. In Fig. 3h the total PL intensity of $\mathrm{CdSe} / \mathrm{ZnSe}$ QDs with a $\mu$-lens printed immediately above them is shown for different positions of the knife edge (empty circles). The distance between the sample and the knife edge was set to $L=325 \mathrm{~mm}$. The blue curve represents the result of fitting an error function to the experimental data and indicates a close-to-Gaussian beam profile. A corresponding Gaussian function with a width of $\sigma=(5.3 \pm 0.2) \mathrm{mm}$ is drawn in the background as 


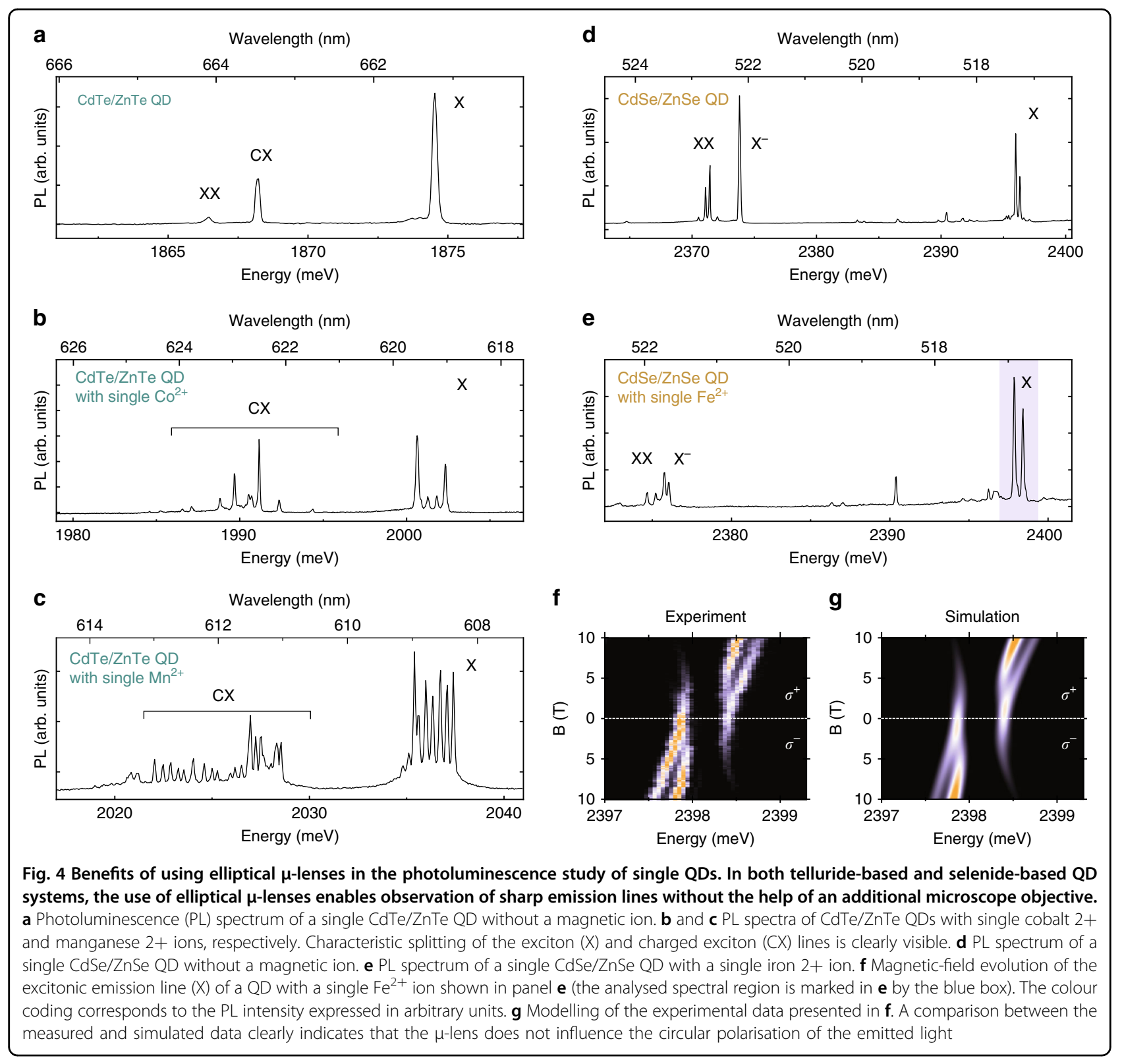

the blue shaded area. This result indicates that the halfangle divergence of the PL beam is 16.4 milliradians $(\theta=$ $0.94^{\circ}$ ), which means that the light emitted from the sample can be collected by an optical setup with an NA as low as 0.016 .

\section{Microlens arrays for solotronic systems}

The CdTe/ZnTe and CdSe/ZnSe QD systems were selected to demonstrate the application of the elliptical $\mu$ lenses in the field of optoelectronics based on solitary dopant-solotronics ${ }^{42,43}$. When doped with single magnetic ions, such QDs can be used to efficiently probe and manipulate the spin of the magnetic ion they contain ${ }^{44-46}$, which otherwise is not optically active. A major difficulty in the study of this type of system is the nondeterministic character of self-assembled growth.

We fabricated regular arrays of $\mu$-lenses on a series of samples with QDs doped with various magnetic ions. In each case, we were able to find a $\mu$-lens printed over a singly doped QD, including a CdTe/ZnTe QD with a single $\mathrm{Co}^{2+}$ ion (Fig. 4b), a CdTe/ZnTe QD with a single $\mathrm{Mn}^{2+}$ ion (Fig. 4c), and a CdSe/ZnSe QD with a single $\mathrm{Fe}^{2+}$ ion (Fig. 4e). For all of them, we clearly observed a characteristic splitting of the exciton line into components corresponding to different spin projections onto the quantisation axis ${ }^{42-44}$. In particular, Fig. 4f displays the magnetic-field evolution of the excitonic emission lines highlighted in Fig. 4e by the blue box. It features a 
characteristic zero-field anticrossing and shows a gradual rise of the polarisation degree due to thermalisation occurring with increasing magnetic field ${ }^{42}$. The results of numerical simulations are shown in Fig. 4g (for simulation details, see Supplementary Information). The direct correspondence between the experimental and simulated data indicates that the use of $\mu$-lenses does not affect the polarisation of the emitted light or its temporal stability.

\section{Discussion}

We demonstrated that elliptical $\mu$-lenses can work over a broad spectral range - at least from 500 to $800 \mathrm{~nm}$-and potentially also at longer wavelengths since the IP-Dip photoresist we used is transparent up to $1610 \mathrm{~nm}^{26}$. We showed that DLW-printed microstructures can be produced not only over optically active objects well protected against environmental conditions (such as QDs) but also on extremely fragile S-TMD-based and h-BN-based heterostructures, whose internal cohesion and adhesion to the substrate are governed by weak, dipole-dipole-like van der Waals interactions.

\section{Perspectives}

Owing to the broad compatibility of the DLW technique, the designed elliptical $\mu$-lenses can be directly applied to a variety of optical systems. An example of such a system potentially benefiting from the $\mu$-lenses is a single-nanowire laser ${ }^{47,48}$. Usually, after the growth process, nanowires are transferred onto a substrate with their longer axis parallel to the substrate surface. Most of the light generated by these lasers is emitted from the point-like nanowire ends, and as a result, one obtains an isotropic far-field pattern. The application of the elliptical $\mu$-lenses could result in this case in an ultra-low-divergence laser beam.

Nanowires also serve as effective detectors of terahertz radiation and as such are used in photoconductive antennas (PCAs) ${ }^{49}$. A device of this type requires pulsed laser illumination focused on the nanowire to generate a sizeable electrical signal. The terahertz radiation is brought to the antenna by a parabolic mirror, and the infrared laser feed is usually provided through a few millimetres in diameter hole in the centre of the mirror. Unfortunately, due to the low NA of the infrared part of the setup, the laser power must be inconveniently high. This issue can be readily solved by using an elliptical $\mu$ lens, enabling the use of standard focusing optics with a small NA to obtain a preliminary focal spot of the infrared laser, which can then be further focused to the single micrometre level.

The limited NA of an optical setup becomes problematic in experiments performed in high magnetic fields where access to the sample is restricted. Moreover, due to inductance issues, one has to avoid metal parts being close to the sample. Similar limitations play an important role in the case of measurements involving microwave radiation, e.g., optically detected magnetic resonance $(\mathrm{ODMR})^{50,51}$ or optically detected nuclear resonance ${ }^{52}$. As microwave cavities are inconvenient for optical measurements of single nanoscale emitters, an elliptical $\mu$-lens could help one focus on the emitter, even with a poorly focused excitation beam, and simultaneously increase the amount of light collected by distant optical elements.

It is worth noting that potential applications of the elliptical $\mu$-lenses are not limited to samples that emit light. Powerful optical techniques, such as Raman scattering, can also benefit from the results presented in this paper. In measurements of this type, the delivery of excitation laser light with sufficient power density to the sample surface is as important as efficient collection of the scattered light, whose intensity is typically very low. Again, as Raman scattering is usually isotropic, utilisation of the elliptical $\mu$-lenses can increase the available WD and substantially decrease the required NA of the collection optics. It is important to note that the Raman signal of the $\mu$-lens itself should not obscure the signal from the sample. The photoresist used in this article (IP-Dip Nanoscribe GmbH) does not exhibit any Raman scattering response in two spectral regions: $1800-2850 \mathrm{~cm}^{-1}$ and above $3115 \mathrm{~cm}^{-1}$. This does not mean, however, that other parts of the electromagnetic spectrum are totally inaccessible. One must keep in mind that since the highest intensity of the laser light is concentrated on the sample surface and not inside the $\mu$-lens volume, the Raman scattering signal from the photoresist is relatively weak (see Supplementary Information for details) and does not significantly affect the Raman spectrum of the sample.

A combination of the above functionalities offered by the elliptical $\mu$-lenses might also be useful for integrated photonic and microfluidic circuits, e.g., opto-fluidic chips $^{53,54}$. The design and fabrication of these devices have recently become remarkably advanced ${ }^{55,56}$. Their proper operation requires focusing a single or multiple laser beams with micrometre precision and collecting the emitted optical signal. With the elliptical $\mu$-lenses, one could use small-NA and long-WD optics to simultaneously achieve both of these goals.

Finally, the elliptical $\mu$-lenses can be used in devices or circuits based on optical fibres, which are still the most common means of introducing light to and collecting it from integrated chips. Such lenses can actually serve as effective coupling elements after minor modification of their design. If the centre of the lens ellipsoid is shifted away from the sample surface (see Supplementary Information), the beam is focused at a few tens of micrometres above the sample. The width of the focal spot can be precisely tuned so that a good match between the distribution of light leaving the ellipsoid and the field mode 
of an optical fibre can be achieved. Such a modified $\mu$-lens combined with a DLW-printed optical fibre microconnector $^{57}$ can be directly implemented on an integrated chip.

We have shown that elliptical $\mu$-lenses, apart from boosting the efficiency of light extraction from and delivery to single quantum emitters, shape the PL light into an ultra-low-divergence beam. Unlike previous approaches, the $\mu$-lenses are capable of collecting the emitted light without the use of additional high-NA objectives. We have demonstrated the $\mu$-lens compatibility with a variety of semiconductor light-emitting systems, including monolayers of transition metal dichalcogenides and self-assembled QDs. We have shown that 3D DLW-based fabrication of micro- and nanostructures on fragile van der Waals heterostructures is possible. Two $\mu$-lens printing strategies have been demonstrated: (i) deterministic, for when the objects to be equipped with $\mu$-lenses are well visible or their locations in/on the substrate are precisely known, and (ii) nondeterministic, suitable when the substrate contains a large number of randomly distributed light emitters, among which only some possess desirable properties (such as a single magnetic ion inside a QD). Our results pave the way for new ultra-long-working-distance optical measurements of micro- and nano-objects not achievable thus far with a standard spectroscopy technique requiring bulky, high-NA objectives.

\section{Materials and methods Microlens fabrication}

Microlenses were fabricated by 3D additive DLW using a two photon photolithography workstation (Photonic Professional, Nanoscribe $\mathrm{GmbH}$ ). A near-infrared femtosecond laser beam was focused inside a droplet of UVcurable photoresist, acting simultaneously as an immersion medium for a microscope objective (dip-in laser lithography-DiLL). Only inside the focal spot volume was the intensity of the laser light high enough for twophoton polymerisation (TPP) to occur. The smallest possible volume to be solidified, called a voxel, was an ellipsoid of revolution of size $\sim 0.4 \mu \mathrm{m}$ in the plane perpendicular to the laser beam and $1.2 \mu \mathrm{m}$ along the beam. When fabricating the elliptical $\mu$-lenses, we used a $\times 100$, $\mathrm{NA}=1.3$ (Zeiss) immersion objective and a negative-tone photoresist (IP-Dip, Nanoscribe $\mathrm{GmbH}$ ). The relative position of a voxel with respect to the sample was changed with the aid of a piezoelectric XYZ stage. After the exposure, the resist was developed for $20 \mathrm{~min}$ in a bath of propylene glycol methyl ether acetate (PGMEA) stirred at $150 \mathrm{rpm}$, then washed for $20 \mathrm{~min}$ in isopropanol (IPA) stirred at $150 \mathrm{rpm}$ and finally gently rinsed with fresh isopropanol.

To speed up the DLW printing, the trajectory of the laser inside the resist was programmed manually without any slicing software. The lens was printed layer by layer, and the voxels followed a spiral path in the clockwise and counterclockwise directions alternately. To preserve a smooth shape of the ellipsoid, a three-fold exposure of its outer shell with decreased laser power was performed, and the vertical spacing between the layers was adjusted to the local curvature of the lens.

The choice of IP-Dip resist was dictated by the fact that it maintains its mechanical and optical properties down to cryogenic temperatures ${ }^{57}$. Other resists, however, with different optical and mechanical properties can also be used in the DLW process ${ }^{58,59}$. The PL data along with the transmission and Raman scattering spectra of the IP-Dip (Nanoscribe $\mathrm{GmbH}$ ) negative-tone photoresist are presented in the Supplementary Information. The details of the $\mu$-lens printing procedure are also given there.

\section{Samples}

Single layers of $\mathrm{MoSe}_{2}$ and $\mathrm{WSe}_{2}$ (bulk crystals purchased from HQ Graphene) were mechanically exfoliated with Microworld F07 tape and transferred by all-dry viscoelastic stamping ${ }^{60}$ onto $\mathrm{SiO}_{2}(90 \mathrm{~nm}) / \mathrm{Si}$ substrates. After that, all samples were covered with exfoliated, 3-30 nm-thick h-BN flakes acting as a protective layer. The samples were annealed at $180^{\circ} \mathrm{C}$ for $20 \mathrm{~min}$ immediately after the transfer to reduce the amount of air bubbles formed between the constituent layers. Capping the samples with h-BN was necessary to prevent their degradation during the photoresist development. However, there are photoresists that do not influence the quality of S-TMD samples ${ }^{17,61}$. The assembled van der Waals heterostructures were additionally annealed in air for $1 \mathrm{~h}$ at $180^{\circ} \mathrm{C}$ just before the 3D printing.

Samples with self-assembled QDs were prepared by a molecular beam epitaxy (MBE) technique. More details on the growth procedures of $\mathrm{CdTe} / \mathrm{ZnSe}$ and $\mathrm{CdTe} / \mathrm{ZnTe}$ QDs with and without magnetic ions can be found in refs. ${ }^{40,42,43}$.

\section{PL measurements}

PL measurements were performed with diode lasers of three different wavelengths: $\lambda=405 \mathrm{~nm}$ for $\mathrm{CdSe} / \mathrm{ZnSe}$ samples, $\lambda=532 \mathrm{~nm}$ for $\mathrm{CdTe} / \mathrm{ZnTe}$ samples and $\lambda=647 \mathrm{~nm}$ for S-TMD samples. While measuring the enhancement of the PL intensity for the S-TMD-based heterostructures, the power of the laser beam focused on the sample by a single lens with a 1-inch diameter and $f=500 \mathrm{~mm}$ (without any additional microscope objectives) was kept at $24 \mu \mathrm{W}$. The emitted light was dispersed in a $75 \mathrm{~cm}$ spectrometer (Acton SP2750) equipped with a charge-coupled device (CCD) camera. In each measurement, two PL spectra were taken with the same acquisition parameters: a background spectrum from a spot on the sample where there was only substrate without any 
light emitter and a measured spectrum from a location where a microlens was printed over the light emitter. The final spectrum was obtained as the difference between the measured spectrum and the background spectrum. During PL measurements of single QDs, the samples were placed in an optical cryostat featuring a $10 \mathrm{~T}$ superconducting magnet. These measurements were performed in a pumped helium bath $(1.6 \mathrm{~K})$ with standard polarisation optics in the detection path.

The evaluation of the PL intensity enhancement for the QDs required taking into account the fact that without a $\mu$-lens, the observed PL spectrum, which seemingly did not feature any narrow peaks, was in fact composed of many sharp emission lines coming from individual QDs within the perimeter of the excitation laser spot. Therefore, the PL intensity gain could be estimated as $G=I_{1 \mathrm{QD}}^{\Delta \lambda}\left(I_{1 \mathrm{QD}}^{\Delta \lambda}\right)^{-1} N_{\mathrm{QDs}}^{\Delta \lambda}$, where $I^{\Delta \lambda}{ }_{1 \mathrm{QD}}$ is the intensity of a sharp emission line coming from a given QD under the elliptical $\mu$-lens integrated over the spectral range $\Delta \lambda$, $I^{\Delta \lambda}{ }_{\mathrm{QDs}}$ corresponds to the PL intensity obtained in the same location on the sample integrated over the same spectral range $\Delta \lambda$ but without the $\mu$-lens, and finally, $N^{\Delta l}{ }_{\text {QDs }}$ represents the number of QDs contributing to the PL spectrum in the spectral range $\Delta \lambda$. $N^{\Delta \lambda}{ }_{\mathrm{QDs}}$ can be expressed as $\rho_{\mathrm{QDs}} A I^{\Delta \lambda}{ }_{\mathrm{QDs}}\left(I_{\mathrm{QDs}}^{\mathrm{tot}}\right)^{-1}$, where $\rho_{\mathrm{QDs}}$ is the lateral density of QDs, equal in our case to approximately $10^{9}-10^{10} \mathrm{~cm}^{-2}, A$ is the area of the laser spot, and $I^{\mathrm{tot}}$ QDs represents the intensity of the QD PL spectrum acquired without the $\mu$-lens integrated over the whole spectral range. The size of the excitation beam spot at the sample position was measured with a Coherent BeamMaster profiler. The value of the PL intensity gain factor $G$ obtained in the course of this analysis was on the order of 100 .

\section{Travelling knife-edge measurements}

To perform travelling knife-edge measurements of the PL beam size and divergence, a motorised knife-edge was placed $325 \mathrm{~mm}$ from the sample. The outgoing light was introduced into a $50 \mu \mathrm{m}$-diameter step-index multimode optical fibre, whose NA was equal to 0.22 , with the aid of a lens of $f=200 \mathrm{~mm}$ and diameter $d=70 \mathrm{~mm}$ positioned $350 \mathrm{~mm}$ from the sample. After filtering the laser light out with a $488 \mathrm{~nm}$ long-pass dichroic filter, the outgoing light was fed into a $75 \mathrm{~cm}$ spectrometer (Acton SP2750) equipped with a CCD camera. A diffuser plate with Lambertian scattering characteristics was placed in front of the optical fibre surface to guarantee the same intensity of light arriving at different angles. The excitation laser beam was introduced onto the optical axis with a longpass, 2-inch $\times 2$-inch dichroic filter placed $175 \mathrm{~mm}$ from the sample. To measure the NA of the experimental setup, a VRC1 detection card from Thorlabs acting as an isotropic light source was used in place of the sample. The acceptance angle of the setup was only limited by the diameter of the collection lens and was approximately five times the measured divergence of the PL beam coming from the printed $\mu$-lens.

\section{Numerical simulations}

The elliptical $\mu$-lens performance was simulated within the formalism of the FDTD method for solving Maxwell's equations with the FDTD Solutions software provided by Lumerical Inc. The 3D simulation box contained a point dipole light source $(\lambda=633 \mathrm{~nm})$ positioned $50 \mathrm{~nm}$ below the surface of a substrate made of an $n=3$ material and capped with an ellipsoid of revolution made of a material with $n=1.53$. The lengths of the ellipsoid half-axes and the position with respect to the substrate surface are given by Eqs. (1) and (2). The simulation box $\left(20 \times 20 \times 21 \mu \mathrm{m}^{3}\right)$ consisted of more than $2.6 \times 10^{8}$ Yee cells and was limited by perfectly matched boundary layers at the top and bottom and by layers satisfying symmetric or antisymmetric boundary conditions (depending on the dipole orientation) at the sides. The electric field was recorded on a set of screens surrounding the lens at the top and the sides, and a built-in farfieldexact function was used to calculate the far-field light intensity for the three cardinal dipole orientations. Their incoherent sum represented the total far-field radiation distribution. It was then integrated over the emission angle and normalised to give the collected power of the emitted light as a function of the collection half-angle. The simulations were also carried out for a half-spherical lens and a bare substrate.

Ray-tracing simulations were performed with custom software written in Python 3.7 featuring the full form of the Fresnel equations and taking into account recursive reflections at the interfaces between different materials in the 3D simulation box.

\section{Acknowledgements \\ This work was supported by the National Science Centre of Poland (NCN) Grants 2016/21/N/ST3/03379, 2015/18/E/ST3/00559 and 2019/03/X/ST3/01969, the EU Graphene Flagship project (No. 785219) and by the ATOMOPTO project (TEAM programme of the Foundation for Polish Science cofinanced by the EU within the ERD Fund). A.R. was funded by the Polish Ministry of Science and Higher Education as part of the "Diamentowy Grant" project for the years 2018-2022. K.W. and T.T. acknowledge support from the Elemental Strategy Initiative conducted by the MEXT, Japan and the CREST (JPMJCR15F3), JST. The photolithography laboratory was funded by the European Regional Development Fund Operational Programme (POIG.02.01.00-14-122/09-00).}

\section{Author details}

${ }^{1}$ Faculty of Physics, University of Warsaw, ul. Pasteura 5, 02-093 Warsaw, Poland. ${ }^{2}$ National Institute for Materials Science, Tsukuba, Ibaraki 305-0044, Japan. ${ }^{3}$ Laboratoire National des Champs Magnétiques Intenses, CNRS-UJF-UPS-INSA, avenue des Martyrs 25, 38042 Grenoble, France

\section{Author contributions}

A.B. conceived the idea underlying the project, analysed spectroscopic data, wrote ray-tracing software and directed the project. Ł.Z. and A.B. designed, optimised and fabricated 3D-printed structures. Ł.Z. performed and analysed 
FDTD simulations. A.B., M.G. and T.K. performed spectroscopic measurements, M.G., W.P., K.N. and A.B. fabricated the samples. K.W. and T.T. grew high-quality h-BN. A.B., M.G., T.K. and A.R. characterised the beam parameters. A.R. performed magnetooptical simulations. J.S. and A.B. characterised the photoresist. A.B., Ł.Z., K.N. and T.K. wrote the manuscript with contributions from all coauthors. P.W. and M.P. participated in discussions. P.K. supervised the project.

\section{Data availability}

The data that support the findings of this study for modelling are available from the corresponding author on reasonable request.

\section{Code availability}

The code for modelling is available from the corresponding author on reasonable request.

\section{Conflict of interest}

The authors declare that they have no conflict of interest.

Supplementary information is available for this paper at https://doi.org/ 10.1038/s41377-020-0284-1.

Received: 21 August 2019 Revised: 25 February 2020 Accepted: 5 March 2020

Published online: 27 March 2020

\section{References}

1. Liu, J. et al. A solid-state source of strongly entangled photon pairs with high brightness and indistinguishability. Nat. Nanotechnol. 14, 586-593 (2019).

2. Chen, Y. et al. Highly-efficient extraction of entangled photons from quantum dots using a broadband optical antenna. Nat. Commun. 9, 2994 (2018).

3. Kaganskiy, A. et al. Enhancing the photon-extraction efficiency of sitecontrolled quantum dots by deterministically fabricated microlenses. Opt. Commun. 413, 162-166 (2018).

4. Somaschi, N. et al. Near-optimal single-photon sources in the solid state. Nat. Photonics 10, 340-345 (2016)

5. Cadeddu, D. et al. A fiber-coupled quantum-dot on a photonic tip. Appl. Phys. Lett. 108, 011112 (2016).

6. Liu, S. F. et al. A deterministic quantum dot micropillar single photon source with $>65 \%$ extraction efficiency based on fluorescence imaging method. Sci. Rep. 7, 13986 (2017).

7. Gschrey, M. et al. Highly indistinguishable photons from deterministic quantum-dot microlenses utilizing three-dimensional in situ electron-beam lithography. Nat. Commun. 6, 7662 (2015).

8. Sapienza, L. et al. Nanoscale optical positioning of single quantum dots for bright and pure single-photon emission. Nat. Commun. 6, 7833 (2015).

9. Gazzano, O. et al. Bright solid-state sources of indistinguishable single photons. Nat. Commun. 4, 1425 (2013).

10. Claudon, J. et al. A highly efficient single-photon source based on a quantum dot in a photonic nanowire. Nat. Photonics 4, 174-177 (2010).

11. Fischbach, S. et al. Single quantum dot with microlens and 3D-printed microobjective as integrated bright single-photon source. ACS Photonics 4 1327-1332 (2017)

12. Thiele, S. et al. Ultra-compact on-chip LED collimation optics by 3D femtosecond direct laser writing. Opt. Lett. 41, 3029-3032 (2016).

13. Hsu, H. C. et al. Optimized semi-sphere lens design for high power LED package. Microelectron. Reliabil. 52, 894-899 (2012).

14. Moiseev, M. A., Doskolovich, L. L. \& Kazanskiy, N. L. Design of high-efficient freeform LED lens for illumination of elongated rectangular regions. Opt. Express 19, A225-A233 (2011).

15. Johlin, E. et al. Broadband highly directive 3D nanophotonic lenses. Nat. Commun. 9, 4742 (2018).

16. Sartison, $M$. et al. Combining in-situ lithography with 3D printed solid immersion lenses for single quantum dot spectroscopy. Sci. Rep. 7, 39916 (2017).

17. Woodhead, C. S. et al. Increasing the light extraction and longevity of TMDC monolayers using liquid formed micro-lenses. 2D Mater. 4, 015032 (2016).
18. Schlehahn, A. et al. Generating single photons at gigahertz modulation-speed using electrically controlled quantum dot microlenses. Appl. Phys. Lett. 108 021104 (2016).

19. Assafrao, A. C. et al. Application of micro solid immersion lens as probe for near-field scanning microscopy. Appl. Phys. Lett. 104, 101101 (2014).

20. Hu, C. N., Hsieh, H. T. \& Su, G. D. J. Fabrication of microlens arrays by a rolling process with soft polydimethylsiloxane molds. J. Micromech. Microeng. 21, 065013 (2011).

21. Hadden, J. P. et al. Strongly enhanced photon collection from diamond defect centers under microfabricated integrated solid immersion lenses. Appl. Phys. Lett. 97, 241901 (2010).

22. Descartes, R. La dioptrique. In: Discours de la méthode pour bien conduire sa raison et chercher la vérité dans les sciences, plus la dioptrique, les météores et la géométrie, vol. Discours Huictiesme, 89-121 (A Leyde de l'Imprimerie de Jan Maire, Leyde, 1637).

23. Dottermusch, $\mathrm{S}$. et al. Exposure-dependent refractive index of nanoscribe ipdip photoresist layers. Opt. Lett. 44, 29-32 (2019).

24. Schell, A. W., Neumer, T. \& Benson, O. Numerical analysis of efficient light extraction with an elliptical solid immersion lens. Opt. Lett. 39, 4639-4642 (2014).

25. Yang, J. J., Hugonin, J. P. \& Lalanne, P. Near-to-far field transformations for radiative and guided waves. ACS Photonics 3, 395-402 (2016).

26. Dietrich, P. I. et al. In situ 3D nanoprinting of free-form coupling elements for hybrid photonic integration. Nat. Photonics 12, 241-247 (2018).

27. Thiele, S. et al. 3D-printed eagle eye: compound microlens system for foveated imaging. Sci. Adv. 3, e1602655 (2017).

28. Dietrich, P. I. et al. Printed freeform lens arrays on multi-core fibers for highly efficient coupling in astrophotonic systems. Opt. Express 25, 18288-18295 (2017).

29. Gissibl, T. et al. Two-photon direct laser writing of ultracompact multi-lens objectives. Nat. Photonics 10, 554-560 (2016).

30. Gissibl, T. et al. Sub-micrometre accurate free-form optics by threedimensional printing on single-mode fibres. Nat. Commun. 7, 11763 (2016).

31. Wang, Q. H. et al. Electronics and optoelectronics of two-dimensional transition metal dichalcogenides. Nat. Nanotechnol. 7, 699-712 (2012).

32. Geim, A. K. \& Grigorieva, I. V. Van der Waals heterostructures. Nature 499 419-425 (2013).

33. Binder, J. et al. Upconverted electroluminescence via Auger scattering of interlayer excitons in van der Waals heterostructures. Nat. Commun. 10, 2335 (2019).

34. Lyons, T. P. et al. The valley Zeeman effect in inter- and intra-valley trions in monolayer WSe, Nat. Commun. 10, 2330 (2019).

35. Mak, K. F., Xiao, D. \& Shan, J. Light-valley interactions in 2D semiconductors. Nat. Photonics 12, 451-460 (2018).

36. Yong, C. K. et al. Biexcitonic optical stark effects in monolayer molybdenum diselenide. Nat. Phys. 14, 1092-1096 (2018).

37. Sun, Z. P., Martinez, A. \& Wang, F. Optical modulators with 2D layered materials. Nat. Photonics 10, 227-238 (2016).

38. Mak, K. F. \& Shan, J. Photonics and optoelectronics of 2D semiconductor transition metal dichalcogenides. Nat. Photonics 10, 216-226 (2016).

39. Kazimierczuk, T. et al. Magnetophotoluminescence study of intershell exchange interaction in CdTe/ZnTe quantum dots. Phys. Rev. B 84, 165319 (2011).

40. Kobak, J. et al. Comparison of magneto-optical properties of various excitonic complexes in CdTe and CdSe self-assembled quantum dots. J. Phys.: Condens. Matter 28, 265302 (2016).

41. Khosrofian, J. M. \& Garetz, B. A. Measurement of a Gaussian laser beam diameter through the direct inversion of knife-edge data. Appl. Opt. 22 3406-3410 (1983).

42. Smoleński, T. et al. Magnetic ground state of an individual $\mathrm{Fe}^{2+}$ ion in strained semiconductor nanostructure. Nat. Commun. 7, 10484 (2016).

43. Kobak, J. et al. Designing quantum dots for solotronics. Nat. Commun. 5, 3191 (2014).

44. Besombes, L. et al. Probing the spin state of a single magnetic ion in an individual quantum dot. Phys. Rev. Lett. 93, 207403 (2004).

45. Le Gall, $\mathbf{C}$. et al. Optical spin orientation of a single manganese atom in a semiconductor quantum dot using quasiresonant photoexcitation. Phys. Rev. Lett. 102, 127402 (2009).

46. Goryca, M. et al. Optical manipulation of a single $\mathrm{Mn}$ spin in a CdTe-based quantum dot. Phys. Rev. Lett. 103, 087401 (2009).

47. Evans, T. J. et al. Continuous-wave lasing in cesium lead bromide perovskite nanowires. Adv. Opt. Mater. 6, 1700982 (2018). 
48. Alanis, J. A. et al. Large-scale statistics for threshold optimization of optically pumped nanowire lasers. Nano Lett. 17, 4860-4865 (2017)

49. Peng, K. et al. Single nanowire photoconductive terahertz detectors. Nano Lett. 15, 206-210 (2015)

50. Mao, S. et al. Experimental setup for investigation of nanoclusters at cryogenic temperatures by electron spin resonance and optical spectroscopies. Rev. Sci. Instrum. 85, 073906 (2014).

51. Shinar, J. Optically detected magnetic resonance studies of luminescencequenching processes in $\pi$-conjugated materials and organic light-emitting devices. Laser Photonics Rev. 6, 767-786 (2012).

52. Glenn, D. R. et al. High-resolution magnetic resonance spectroscopy using a solid-state spin sensor. Nature 555, 351-354 (2018).

53. Shi, Y. Z. et al. Nanometer-precision linear sorting with synchronized optofluidic dual barriers. Sci. Adv. 4, eaao0773 (2018).

54. Lee, K. S. et al. An automated Raman-based platform for the sorting of live cells by functional properties. Nat. Microbiol. 4, 1035-1048 (2019).

55. Sun, C. et al. Single-chip microprocessor that communicates directly using light. Nature 528, 534-538 (2015).
56. Shi, Y. Z. et al. Sculpting nanoparticle dynamics for single-bacteria-leve screening and direct binding-efficiency measurement. Nat. Commun. 9, 815 (2018).

57. Bogucki, A. et al. Optical fiber micro-connector with nanometer positioning precision for rapid prototyping of photonic devices. Opt. Express $\mathbf{2 6}$ 11513-11518 (2018).

58. Cicha, K. et al. Young's modulus measurement of two-photon polymerized micro-cantilevers by using nanoindentation equipment. J. Appl. Phys. 112 094906 (2012).

59. Ovsianikov, A. et al. Ultra-low shrinkage hybrid photosensitive material for two-photon polymerization microfabrication. ACS Nano 2, 2257-2262 (2008).

60. Castellanos-Gomez, A. et al. Deterministic transfer of two-dimensional materials by all-dry viscoelastic stamping. 2D. Materials 1, 011002 (2014).

61. Higgins, D. A. et al. High-resolution direct-write multiphoton photolithography in poly(methylmethacrylate) films. Appl. Phys. Lett. 88, 184101 (2006). 